\title{
Extremal graphs for vertex-degree-based invariants with given degree sequences *
}

\author{
Muhuo $\mathrm{Liu}^{1,2}$, Kexiang $\mathrm{Xu}^{3}$, Xiao-Dong Zhang ${ }^{4}$, \\ ${ }^{1}$ Department of Mathematics, College of Mathematics and Informatics, \\ South China Agricultural University, Guangzhou, 510642, China \\ ${ }^{2}$ College of Mathematics and Statistics, Shenzhen University, \\ Shenzhen, 518060, China \\ ${ }^{3}$ College of Science, Nanjing University of Aeronautics \& Astronautics \\ Nanjing, Jiangsu, 210016, PR China \\ ${ }^{4}$ School of Mathematical Sciences, MOE-LSC and SHL-MAC, \\ Shanghai Jiao Tong University, 800 Dongchuan road, Shanghai, 200240, P. R. China
}

\begin{abstract}
For a symmetric bivariable function $f(x, y)$, let the connectivity function of a connected graph $G$ be $M_{f}(G)=\sum_{u v \in E(G)} f(d(u), d(v))$, where $d(u)$ is the degree of vertex $u$. In this paper, we prove that for an escalating (de-escalating) function $f(x, y)$, there exists a BFS-graph with the maximum (minimum) connectivity function $M_{f}(G)$ among all graphs with a $c$-cyclic degree sequence $\pi=\left(d_{1}, d_{2}, \ldots, d_{n}\right)$ and $d_{n}=1$, and obtain the majorization theorem for connectivity function for unicyclic and bicyclic degree sequences. Moreover, some applications of graph invariants based on degree are included.
\end{abstract}

Keywords: Connectivity function; c-cyclic graph; degree sequence; majorization; BFS-graph.

\section{Introduction}

Throughout this paper, $G$ denotes a simple connected graph with $n$ vertices and $m$ edges, unless specified otherwise. If $m=n+c-1$, then $G$ is called a c-cyclic graph. In particular, when $c=0,1$ or 2 , then $G$ is called a tree, unicyclic graph or bicyclic graph, respectively. As usual, denote $N_{G}(v)$ the neighbor set of vertex $v$ in $G$, let $d_{G}(v)$ be the degree of $v$, and let $N_{G}[v]=N_{G}(v) \cup\{v\}$. When there is no confusion,

\footnotetext{
* The first author is supported by NSFC of China (No. 11571123), the Training Program for Outstanding Young Teachers in University of Guangdong Province (No. YQ2015027), Guangdong Engineering Research Center for Data Science (No. 2017A-KF02) and Guangdong Province Ordinary University Characteristic Innovation Project (No. 2017KTSCX020). The second author is supported by NNSF of China (No. 11671202) and Chinese Excellent Overseas Researcher Funding in 2016. The third author is supported by the Joint NSFC-ISF Research Program (jointly funded by the National Natural Science Foundation of China and the Israel Science Foundation (No. 11561141001)) and the National Natural Science Foundation of China (No. 11531001).

E-mail addresses: liumuhuo@163.com (M. Liu), kexxu1221@126.com(K. Xu), xiaodong@sjtu.edu.cn (X.-D. Zhang, Corresponding author)
} 
we simplify $N_{G}(v), N_{G}[v]$ and $d_{G}(v)$ as $N(v), N[v]$ and $d(v)$, respectively. If $d(v)=1$, then $v$ is called a pendant vertex. The nonnegative integer sequence $\pi=\left(d_{1}, d_{2}, \ldots, d_{n}\right)$ is called the degree sequence of $G$ if $d_{i}=d\left(v_{i}\right)$ holds for $1 \leq i \leq n$, where $V(G)=\left\{v_{1}, v_{2}, \ldots, v_{n}\right\}$. Throughout this paper, we use $d_{i}$ to denote the $i$-th largest degree of $G$ and we suppose that $d\left(v_{i}\right)=d_{i}$, where $1 \leq i \leq n$. Let $\Gamma(\pi)$ be the class of connected graphs with degree sequence $\pi$.

Among those vertex-degree-based graph invariants, the Randić index $R(G)[13$ and second Zagreb index $M_{2}(G)$ [4] are two famous topological indices, where

$$
R(G)=\sum_{u v \in E(G)}(d(u) d(v))^{-\frac{1}{2}}, \quad \text { and } \quad M_{2}(G)=\sum_{u v \in E(G)}(d(u) d(v)) .
$$

Bollobás and Erdős [1] generalized the concepts of Randić index and second Zagreb index to the general Randić index $W_{\alpha}(G)$, where

$$
W_{\alpha}(G)=\sum_{u v \in E(G)}(d(u) d(v))^{\alpha} .
$$

Similarly with the general Randic index of $G$, the general sum-connectivity index 24$] \chi_{\alpha}(G)$ of $G$ is constructed as :

$$
\chi_{\alpha}(G)=\sum_{u v \in E(G)}(d(u)+d(v))^{\alpha}
$$

where $\chi_{-\frac{1}{2}}(G)$ is known as the sum-connectivity index of $G$ [23], $\chi_{2}(G)$ is also called the third Zagreb index of $G$ [14], while $2 \chi_{-1}(G)$ is equal to the harmonic index of $G$ [3]. The reformulated Zagreb index $Z_{2}(G)$ of $G 12$ is a slight modification of $\chi_{2}(G)$, where

$$
Z_{2}(G)=\sum_{u v \in E(G)}(d(u)+d(v)-2)^{2} .
$$

Another famous vertex-degree-based graph invariants, called the Atom-Bond connectivity index of $G$ [2, is defined as follows:

$$
A B C(G)=\sum_{u v \in E(G)} \sqrt{\frac{d(u)+d(v)-2}{d(u) d(v)}} .
$$

For more results on vertex-degree-based graph invariants, one can refer to 5,18 . In order to study on such graph invariants based on adjacency vertex degree, Wang [16] recently proposed a new symmetric function.

A symmetric bivariate function $f(x, y)$ defined on positive real numbers is called escalating (deescalating) if

$$
f\left(x_{1}, x_{2}\right)+f\left(y_{1}, y_{2}\right) \geq(\text { resp. } \leq) f\left(x_{2}, y_{1}\right)+f\left(x_{1}, y_{2}\right)
$$


holds for any $x_{1} \geq y_{1}>0$ and $x_{2} \geq y_{2}>0$, and the inequality in 1.1 is strict if $x_{1}>y_{1}$ and $x_{2}>y_{2}$. Furthermore, an escalating (de-escalating) function $f(x, y)$ is called a good escalating (de-escalating) function, if $f(x, y)$ satisfies

$$
\frac{\partial f(x, y)}{\partial x}>0, \quad \frac{\partial^{2} f(x, y)}{\partial x^{2}} \geq 0
$$

and

$$
f\left(x_{1}+1, x_{2}\right)+f\left(x_{1}+1, y_{1}-1\right) \geq f\left(x_{2}, y_{1}\right)+f\left(x_{1}, y_{1}\right)
$$

holds for any $x_{1} \geq y_{1}$ and $x_{2} \geq 1$.

Further, Wang 16 defined the connectivity function of a connected graph $G$ associated with a symmetric bivariate function $f(x, y)$ to be

$$
M_{f}(G)=\sum_{u v \in E(G)} f(d(u), d(v))
$$

For a connected graph $G$, let us use the notation $h_{G}(v)$ to denote the distance between $v$ and $v_{1}$, and let $\mathcal{A}_{i}(G)=\left\{v: h_{G}(v)=i\right.$ and $\left.v \in V(G)\right\}$. Then, $\mathcal{A}_{0}(G)=\left\{v_{1}\right\}$. If there is no confusion, we simply write $h(v)$ and $\mathcal{A}_{i}$ in place of $h_{G}(v)$ and $\mathcal{A}_{i}(G)$, respectively.

Definition 1.1 Let $G$ be a connected graph. If there exists an ordering $v_{1} \prec v_{2} \prec \cdots \prec v_{n}$ of $V(G)$ satisfying the following $(i)$ and (ii), then $G$ is called a BFS-graph (see [21]):

(i) $d\left(v_{1}\right) \geq d\left(v_{2}\right) \geq \cdots \geq d\left(v_{n}\right)$ and $h\left(v_{1}\right) \leq h\left(v_{2}\right) \leq \cdots \leq h\left(v_{n}\right)$.

(ii) let $v \in N(u) \backslash N(w), z \in N(w) \backslash N(u)$ such that $h(u)=h(w)=h(v)-1=h(z)-1$, if $u \prec w$, then $v \prec z$.

In some literatures, a $B F S$-tree is also called a greedy tree (see 16, 22]). It is well-known that the $B F S$-tree is unique for any given tree degree sequence $\pi$ 21].

In [16], Wang proved the following general important result.

Theorem 1.1 16] For an escalating function $f(x, y)$, the connectivity function $W_{f}(T)$ is maximized by the greedy tree among trees with given degree sequence.

Later, Zhang et al. 22 studied relations between the extremal trees of two different degree sequences. In order to state their results, we need the following notation. Let $\pi=\left(x_{1}, x_{2}, \ldots, x_{n}\right)$ and $\pi^{\prime}=\left(x_{1}^{\prime}, x_{2}^{\prime}, \ldots, x_{n}^{\prime}\right)$ be two different non-increasing sequences of nonnegative real numbers, we write $\pi \triangleleft \pi^{\prime}$ if and only if $\pi \neq \pi^{\prime}$, 
$\sum_{i=1}^{n} x_{i}=\sum_{i=1}^{n} x_{i}^{\prime}$, and $\sum_{i=1}^{j} x_{i} \leq \sum_{i=1}^{j} x_{i}^{\prime}$ for all $j=1,2, \ldots, n$. Such an ordering is sometimes called majorization ( for example, see 11]). Zhang et al. in [22 proved the following majorization theorem:

Theorem 1.2 22 Given two tree degree sequences $\pi$ and $\pi^{\prime}$ and an escalating function with $\frac{\partial f(x, y)}{\partial x}>0$, and $\frac{\partial^{2} f(x, y)}{\partial x^{2}} \geq 0$, let $T_{\pi}^{*}$ and $T_{\pi^{\prime}}^{*}$ be the two trees with the maximum connectivity function $M_{f}(T)$ with degree sequences $\pi$ and $\pi^{\prime}$, respectively. If $\pi \triangleleft \pi^{\prime}$, then $M_{f}\left(T_{\pi}^{*}\right) \leq M_{f}\left(T_{\pi^{\prime}}^{*}\right)$.

Motivated by the above results, we continued to study on the properties of connectivity function for (good) escalating or de-escalating functions for $c$-cyclic graphs.

The rest of this paper is organized as follows. In Section 2, we proved that for an escalating (deescalating) function $f(x, y)$, there exists a BFS-graph $G$ with the maximum (minimum) connectivity function $M_{f}(G)$ among all graphs with a $c$-cyclic degree sequence $\pi=\left(d_{1}, d_{2}, \ldots, d_{n}\right)$ and $d_{n}=1$. In Section 3, the majorization theorem for connectivity function is obtained for a good escalating function for tree, unicyclic and bicyclic degree sequences. In Section 4, the properties of some graph invariants based on degree are obtained.

\section{The extremal graphs of $\Gamma(\pi)$}

Hereafter, we call $G$ an extremal graph of some graph category $\mathcal{G}$ if either $M_{f}(G)$ is maximized in $\mathcal{G}$ when $f(x, y)$ is escalating or $M_{f}(G)$ is minimized in $\mathcal{G}$ when $f(x, y)$ is de-escalating.

Let $G-u v$ (respectively, $G-u$ ) denote the graph obtained from $G$ by deleting the edge $u v \in E(G)$ (respectively, vertex $u$ and the edges incident with it). Similarly, $G+u v$ is a graph obtained from $G$ by adding an edge $u v \notin E(G)$.

Lemma 2.1 Let $G=(V, E)$ be a connected graph with $u_{1} w_{1} \in E, u_{2} w_{2} \in E, w_{1} w_{2} \notin E$ and $u_{1} u_{2} \notin E$, $d\left(w_{1}\right) \geq d\left(u_{2}\right)$ and $d\left(w_{2}\right) \geq d\left(u_{1}\right)$. Let $G^{\prime}=G-u_{1} w_{1}-u_{2} w_{2}+w_{1} w_{2}+u_{1} u_{2}$.

(i) If $f(x, y)$ is escalating, then $M_{f}\left(G^{\prime}\right) \geq M_{f}(G)$, where $M_{f}\left(G^{\prime}\right)>M_{f}(G)$ if and only if $d\left(w_{1}\right)>$ $d\left(u_{2}\right)$ and $d\left(w_{2}\right)>d\left(u_{1}\right)$.

(ii) If $f(x, y)$ is de-escalating, then $M_{f}\left(G^{\prime}\right) \leq M_{f}(G)$, where $M_{f}\left(G^{\prime}\right)<M_{f}(G)$ if and only if $d\left(w_{1}\right)>$ $d\left(u_{2}\right)$ and $d\left(w_{2}\right)>d\left(u_{1}\right)$.

Proof. By 1.2 and $f\left(d\left(u_{1}\right), d\left(u_{2}\right)\right)=f\left(d\left(u_{2}\right), d\left(u_{1}\right)\right)$, we have

$$
M_{f}\left(G^{\prime}\right)-M_{f}(G)=f\left(d\left(w_{1}\right), d\left(w_{2}\right)\right)+f\left(d\left(u_{2}\right), d\left(u_{1}\right)\right)-f\left(d\left(u_{2}\right), d\left(w_{2}\right)\right)-f\left(d\left(u_{1}\right), d\left(w_{1}\right)\right) .
$$


Then the results follow from the definitions of escalating and de-escalating functions.

Hereafter, let $P_{u v}$ be a shortest path connecting $u$ and $v$ in $G$, and let $C_{q}$ be a cycle with $q$ vertices.

Lemma 2.2 Suppose $G \in \Gamma(\pi)$, and there exist three vertices $u, v$, $w$ of $G$ such that uv $\in E(G)$, $u w \notin E(G), d(v)<d(w) \leq d(u)$, and $d(u)>d(x)$ for all $x \in N(w)$. Then, $G$ is not an extremal graph of $\Gamma(\pi)$.

Proof. We firstly suppose that $u v \notin P_{u w}$, and we may suppose that $z \in N(w) \cap V\left(P_{u w}\right)$. If $v z \notin E(G)$, then let $G_{1}=G+v z+u w-w z-u v$. Clearly, $G_{1} \in \Gamma(\pi)$. Since $d(u)>d(z)$ and $d(w)>d(v)$, the result follows from Lemma 2.1. Otherwise, $v z \in E(G)$. In this case, since $d(w)>d(v)$, there exists a vertex $w^{\prime} \in N(w)$ such that $w^{\prime} \notin N[v]$ and $w^{\prime} \notin V\left(P_{u w}\right)$. Let $G_{2}=G+v w^{\prime}+u w-w w^{\prime}-u v$. Then, $G_{2} \in \Gamma(\pi)$. Note that $d(u)>d\left(w^{\prime}\right)$ and $d(w)>d(v)$. Thus, the result follows from Lemma 2.1.

We secondly consider the case of $u v \in P_{u w}$. In this case, since $d(w)>d(v)$, there exists a vertex $w^{\prime \prime} \in N(w) \backslash V\left(P_{u w}\right)$ such that $w^{\prime \prime} \notin N[v]$. Let $G_{3}=G+v w^{\prime \prime}+u w-w w^{\prime \prime}-u v$. Clearly, $G_{3} \in \Gamma(\pi)$ and hence the result follows from Lemma 2.1.

Lemma 2.3 If $\pi$ is a c-cyclic degree sequence with $c \geq 0$, then there exists an extremal graph $G \in \Gamma(\pi)$ such that $\left\{v_{2}, v_{3}\right\} \subseteq N\left(v_{1}\right)$.

Proof. Let $G$ be an extremal graph of $\Gamma(\pi)$. If $v_{1} v_{2} \notin E(G)$, then there is some vertex $v$ such that $v_{1} v \in E(G)$ and $d\left(v_{1}\right) \geq d\left(v_{2}\right)>d(v)$ and $d\left(v_{1}\right)>d(x)$ holds for all $x \in N\left(v_{2}\right)$, which contradicts Lemma 2.2. Thus, $v_{1} v_{2} \in E(G)$. Now, we assume that $v_{1} v_{3} \notin E(G)$. Then, $d\left(v_{3}\right)>d(v)$ holds for every $v \in N\left(v_{1}\right) \backslash\left\{v_{2}\right\}$. By Lemma 2.2, we may assume that there exists some vertex $u \in N\left(v_{3}\right)$ such that $d(u)=d_{1}$. If $u=v_{2}$, the result already holds. Otherwise, $u \neq v_{2}$, and hence $d(u)=d_{1}=d_{2}=d_{3}$.

If $v_{2} \notin V\left(P_{v_{1} v_{3}}\right)$, choose $u_{0} \in N\left(v_{1}\right) \cap V\left(P_{v_{1} v_{3}}\right)$, since $v_{1} \in N\left(u_{0}\right) \backslash N\left(v_{3}\right)$, there must exist some vertex $v_{0} \in N\left(v_{3}\right) \backslash V\left(P_{v_{1} v_{3}}\right)$ such that $v_{0} \notin N\left[u_{0}\right]$. Let $G_{1}=G+v_{1} v_{3}+u_{0} v_{0}-v_{1} u_{0}-v_{3} v_{0}$. By Lemma 2.1 and $G_{1} \in \Gamma(\pi)$, the result already holds. Otherwise, $v_{2} \in V\left(P_{v_{1} v_{3}}\right)$. In this case, $v_{1} \notin P_{v_{2} v_{3}}$ and $d_{1}=d_{2}=d_{3}$. Now, it can be proved similarly with the case $v_{2} \notin V\left(P_{v_{1} v_{3}}\right)$.

Let $N_{G}(v, p)$ be the neighbor set of vertices of $v$ in $G$ with degree at least $p$.

Lemma 2.4 Let $C_{q}$ be a cycle of an extremal graph $G$ of $\Gamma(\pi)$ with $w_{1} w_{2} \in E\left(C_{q}\right)$ and $P=u_{1} \cdots u_{s-1} u_{s}$ being a path connecting $u_{1}$ and $u_{s}$ such that $u_{1} \in V\left(C_{q}\right)$ and $d\left(w_{1}\right) \geq d\left(w_{2}\right)>d\left(u_{s}\right)$. If $d\left(u_{k}\right) \geq d\left(w_{2}\right)$ 
holds for some vertex $u_{k} \in V(P) \backslash\left\{u_{1}\right\}, N\left(w_{1}\right) \cap\left\{u_{k}, u_{k+1}, \ldots, u_{s}\right\}=\varnothing$ and $N\left(w_{2}\right) \cap\left\{u_{k+1}, u_{k+2}, \ldots, u_{s}\right\}=$ $\varnothing$, then there exists an extremal graph $G^{\prime}$ of $\Gamma(\pi)$ such that $P^{\prime}=u_{1} u_{2} \cdots u_{k}$ is a part of one cycle in $G^{\prime}$ with $N_{G}\left(u_{k}, d\left(w_{2}\right)\right) \subseteq N_{G^{\prime}}\left(u_{k}, d\left(w_{2}\right)\right)$ and $N_{G}\left(u_{i}\right)=N_{G^{\prime}}\left(u_{i}\right)$, where $2 \leq i \leq k-1$. Furthermore, every cycle of $G$, which do not contain the edge $w_{1} w_{2}$, is also a cycle of $G^{\prime}$.

Proof. By the hypothesis, we may suppose that $u_{k}$ is the last vertex of $P$ such that $d\left(u_{k}\right) \geq d\left(w_{2}\right)$, namely, $\max \left\{d\left(u_{j}\right): k+1 \leq j \leq s\right\}<d\left(w_{2}\right)$. Since $d\left(u_{s}\right)<d\left(w_{2}\right)$, we have $2 \leq k \leq s-1$. If $P^{\prime}=u_{1} u_{2} \cdots u_{k}$ is a part of some cycle of $G$, then the result already holds by setting $G^{\prime}=G$. Thus, we may suppose that $P^{\prime}=u_{1} u_{2} \cdots u_{k}$ is not a part of any cycle of $G$.

Let $G_{1}=G+w_{1} u_{k}+w_{2} u_{k+1}-w_{1} w_{2}-u_{k} u_{k+1}$. Since $d\left(w_{2}\right) \leq d\left(u_{k}\right)$ and $d\left(w_{1}\right) \geq d\left(w_{2}\right)>d\left(u_{k+1}\right)$, $G_{1}$ is also an extremal graph of $\Gamma(\pi)$ by Lemma 2.1. In this case, $P^{\prime}=u_{1} u_{2} \cdots u_{k}$ is a part of one cycle of $G_{1}$ such that $N_{G}\left(u_{k}, d\left(w_{2}\right)\right) \subseteq N_{G_{1}}\left(u_{k}, d\left(w_{2}\right)\right)$ and $N_{G}\left(u_{i}\right)=N_{G_{1}}\left(u_{i}\right)$, where $2 \leq i \leq k-1$. Furthermore, every cycle of $G$, which do not contain the edge $w_{1} w_{2}$, is also a cycle of $G_{1}$. Thus, the result holds.

Remark 2.1 By an observation to the proof of Lemma 2.4. $u_{1} \in\left\{w_{1}, w_{2}\right\}$ is also permitted and $u_{1} \notin$ $\left\{w_{1}, w_{2}\right\}$ guarantees the existence of an extremal graph $G^{\prime}$ of $\Gamma(\pi)$ such that $P^{\prime}=u_{1} u_{2} \cdots u_{k}$ is a part of one cycle in $G^{\prime}$ with $N_{G}\left(u_{k}, d\left(w_{2}\right)\right) \subseteq N_{G^{\prime}}\left(u_{k}, d\left(w_{2}\right)\right)$ and $N_{G}\left(u_{i}\right)=N_{G^{\prime}}\left(u_{i}\right)$, where $1 \leq i \leq k-1$.

Let $G$ be a connected graph with $X \subseteq V(G)$. Denote by $G[X]$ the subgraph induced by $X$, and denote by $\mathcal{R}(G)$ the base graph obtained from $G$ by recursively deleting pendant vertices of the resultant graph until no pendant vertices remain. If $G$ is a $c$-cyclic graph, it is easy to see that $\mathcal{R}(G)$ is also a $c$-cyclic graph for $c \geq 1$ and $\mathcal{R}(G)=\varnothing$ holds for $c=0$.

Lemma 2.5 Let $\pi$ be a c-cyclic degree sequence. If $c \geq 1$ and $d_{n}=1$, then there exists an extremal graph $G$ of $\Gamma(\pi)$ such that $\left\{v_{1}, v_{2}, v_{3}\right\}$ forms a triangle.

Proof. It suffices to prove the following four claims:

Claim 1. There exists an extremal graph $G$ of $\Gamma(\pi)$ such that $\left\{v_{2}, v_{3}\right\} \subseteq N\left(v_{1}\right)$ and $v_{1}$ lies on a cycle of $G$.

By Lemma 2.3, there is an extremal graph $G \in \Gamma(\pi)$ such that $\left\{v_{2}, v_{3}\right\} \subseteq N\left(v_{1}\right)$. We assume that claim 1 does not hold.

If $v_{1} \notin V(\mathcal{R}(G))$, then there must exist one edge $w_{1} w_{2}$ in a cycle and one vertex $u$ in $N\left(v_{1}\right)$ such that $u \notin V(\mathcal{R}(G))$ and $d(u) \geq d\left(w_{1}\right) \geq d\left(w_{2}\right)$ (since $\left\{v_{2}, v_{3}\right\} \subseteq N\left(v_{1}\right)$ ). By Lemma 2.4 and Remark 2.1, 
there exists an extremal graph $G_{1}$ of $\Gamma(\pi)$ such that $\left\{v_{2}, v_{3}\right\} \subseteq N_{G_{1}}\left(v_{1}\right)$ and $v_{1}$ lies on a cycle of $G_{1}$, a contradiction.

Otherwise, $v_{1} \in V(\mathcal{R}(G))$. Now, since $v_{1}$ does not lie on any cycle of $G, v_{1}$ is a cut vertex and $G-v_{1}$ contains $d_{1}$ components, say $D_{1}, D_{2}, \ldots, D_{d_{1}}$. Since $d_{n}=1$, we may suppose that $v_{n}$ lies on $D_{1}$ and $D_{2}$ is a component containing a cycle (Recall that $v_{1} \in V(\mathcal{R}(G)), G-v_{1}$ contains at least two components containing cycles). In this case, by Lemma 2.4 and Remark 2.1. there exists an extremal graph $G_{2}$ of $\Gamma(\pi)$ such that $\left\{v_{2}, v_{3}\right\} \subseteq N_{G_{2}}\left(v_{1}\right)$ and $v_{1}$ lies on a cycle of $G_{2}$, a contradiction. This completes the proof of Claim 1.

Claim 2. There exists an extremal graph $G$ of $\Gamma(\pi)$ such that $v_{1} v_{2}$ lies on a cycle of $G$ and $\left\{v_{2}, v_{3}\right\} \subseteq$ $N\left(v_{1}\right)$

By Claim 1, we may directly suppose that $G$ is an extremal graph of $\Gamma(\pi)$ such that $\left\{v_{2}, v_{3}\right\} \subseteq N\left(v_{1}\right)$ and $v_{1}$ lies on some cycle $C_{q}$ of $G$. We assume that claim 2 does not hold. Let $d\left(w_{0}\right)=\max \{d(v): v \in$ $\left.V\left(C_{q}\right) \backslash\left\{v_{1}\right\}\right\}$. If $d\left(w_{0}\right) \geq d(v)$ for some vertex $v \in N\left(v_{2}\right) \backslash\left\{v_{1}\right\}$, then $v w \notin E(G)$ and $v_{2} w_{0} \notin E(G)$, where $w \in\left(N\left(w_{0}\right) \cap V\left(C_{q}\right)\right) \backslash\left\{v_{1}\right\}$ (Otherwise, Claim 2 already holds). Let $G_{3}=G+v_{2} w_{0}+v w-w_{0} w-v_{2} v$. Since $d\left(w_{0}\right) \geq d(v)$ and $d\left(v_{2}\right) \geq d(w), G_{3}$ is an extremal graph of $\Gamma(\pi)$ such that Claim 2 holds, a contradiction. Otherwise, $2 \leq d\left(w_{0}\right)<d(v)$ holds for each $v \in N\left(v_{2}\right)$. Let $v^{\prime} \in N(v) \backslash\left\{v_{2}\right\}$ and let $w^{\prime} \in\left(N\left(v_{1}\right) \cap V\left(C_{q}\right)\right) \backslash\left\{v_{3}\right\}$, where $v \in N\left(v_{2}\right) \backslash\left\{v_{1}\right\}$. Since Claim 2 does not hold, $v_{1} v \notin E(G)$ and $v^{\prime} w^{\prime} \notin E(G)$. Let $G_{4}=G+v_{1} v+v^{\prime} w^{\prime}-v_{1} w^{\prime}-v v^{\prime}$. Since $d\left(v_{1}\right) \geq d\left(v^{\prime}\right)$ and $d(v)>d\left(w_{0}\right) \geq d\left(w^{\prime}\right), G_{4}$ is an extremal graph of $\Gamma(\pi)$ such that Claim 2 holds. This completes the proof of Claim 2.

Claim 3. There is an extremal graph $G$ of $\Gamma(\pi)$ such that $v_{1} v_{2}$ and $v_{1} v_{3}$ lie on a cycle of $G$.

By Claim 2, we may directly suppose that $G$ is an extremal graph of $\Gamma(\pi)$ such that $v_{1} v_{2}$ lies on a cycle of $G$ and $\left\{v_{2}, v_{3}\right\} \subseteq N\left(v_{1}\right)$. Choose $u_{0} \in\left(V\left(C_{q}\right) \cap N\left(v_{2}\right)\right) \backslash\left\{v_{1}\right\}$.

If Claim 3 does not holds, then $v_{2} v_{3} \notin E(G), u_{0} v_{3} \notin E(G)$ and there exists $v_{0} \in N\left(v_{3}\right) \backslash\left\{v_{1}\right\}$ such that $v_{0} \notin N\left[u_{0}\right]\left(\right.$ as $\left.d\left(v_{3}\right) \geq d\left(u_{0}\right) \geq 2\right)$, then let $G_{5}=G+v_{2} v_{3}+u_{0} v_{0}-v_{0} v_{3}-u_{0} v_{2}$. By Lemma 2.1. $G_{5}$ is also an extremal graph of $\Gamma(\pi)$ such that $\left\{v_{1}, v_{2}, v_{3}\right\}$ forms a triangle of $G_{5}$, a contradiction. Thus, Claim 3 holds.

Claim 4. There is an extremal graph $G$ of $\Gamma(\pi)$ such that $\left\{v_{1}, v_{2}, v_{3}\right\}$ forms a triangle of $G$.

By Claim 3, we may directly suppose that $G$ is an extremal graph of $\Gamma(\pi)$ such that $\left\{v_{1} v_{2}, v_{1} v_{3}\right\}$ are 
two edges of some cycle of $G$, while $v_{2} v_{3} \notin E(G)$. Furthermore, we may suppose that

$$
q=\min \left\{p, \text { where } v_{1} v_{2} \in E\left(C_{p}\right) \text { and } v_{1} v_{3} \in E\left(C_{p}\right)\right\},
$$

and hence $\left|N\left(v_{2}\right) \cap V\left(C_{q}\right)\right|=\left|N\left(v_{3}\right) \cap V\left(C_{q}\right)\right|=2$. We assume that Claim 4 does not hold. Two cases occur as follows:

Case 1. $d_{2} \geq 3$.

Choose $w_{1} \in\left(N\left(v_{3}\right) \cap V\left(C_{q}\right)\right) \backslash\left\{v_{1}\right\}$. If there exists some vertex $w_{2} \in N\left(v_{2}\right) \backslash V\left(C_{q}\right)$ such that $w_{1} w_{2} \notin$ $E(G)$, then let $G_{6}=G+v_{2} v_{3}+w_{1} w_{2}-w_{2} v_{2}-w_{1} v_{3}$. By Lemma 2.1. $G_{6}$ is also an extremal graph of $\Gamma(\pi)$ such that $\left\{v_{1}, v_{2}, v_{3}\right\}$ forms a triangle in $G_{6}$, and hence Claim 4 holds, a contradiction. Otherwise, $w \in N\left(w_{1}\right)$ holds for each $w \in N\left(v_{2}\right) \backslash V\left(C_{q}\right)$, which implies that $d\left(v_{2}\right)=d\left(v_{3}\right)=d\left(w_{1}\right) \geq 3$.

Nota that $v_{1} w_{1} \notin E(G)$. Otherwise, $\left\{v_{1}, v_{3}, w_{1}\right\}$ forms a triangle of $G$, and hence Claim 4 holds (since $\left.d\left(v_{2}\right)=d\left(v_{3}\right)=d\left(w_{1}\right)\right)$, a contradiction. If there exists some vertex $z \in N\left(v_{1}\right) \backslash\left\{v_{2}, v_{3}\right\}$ such that $z w \notin E(G)$, then let $G_{7}=G+v_{1} w_{1}+w z-w_{1} w-v_{1} z$. By Lemma 2.1, $G_{7}$ is also an extremal graph of $\Gamma(\pi)$ such that $\left\{v_{1}, v_{3}, w_{1}\right\}$ forms a triangle in $G_{7}$, a contradiction. Thus, $z \in N(w)$ holds for each $z \in N\left(v_{1}\right) \backslash\left\{v_{2}, v_{3}\right\}$. Since $w_{1} \in N(w) \backslash N\left(v_{1}\right)$, we have $d\left(v_{1}\right)=d\left(v_{2}\right)=d\left(v_{3}\right)=d\left(w_{1}\right)=d(w) \geq 3$.

In this case, $w v_{3} \notin E(G)$ (Otherwise, Claim 4 already holds). Since $w \in N\left(v_{2}\right) \backslash N\left(v_{3}\right)$ and $d\left(v_{2}\right)=$ $d\left(v_{3}\right)$, there exists some vertex $z^{\prime} \in N\left(v_{3}\right) \backslash V\left(C_{q}\right)$ such that $v_{2} z^{\prime} \notin E(G)$. Let $G_{8}=G+v_{3} w+v_{2} z^{\prime}-$ $v_{2} w-v_{3} z^{\prime}$. Since $d\left(v_{1}\right)=d\left(v_{2}\right)=d\left(v_{3}\right)=d\left(w_{1}\right)=d(w)$, by Lemma 2.1. $G_{8}$ is also an extremal graph of $\Gamma(\pi)$ such that $\left\{v_{3}, w_{1}, w\right\}$ forms a triangle of $G_{8}$, contrary with $d\left(v_{1}\right)=d\left(v_{2}\right)=d\left(v_{3}\right)=d\left(w_{1}\right)=d(w)$.

Case 2. $d_{2}=2$.

Suppose $P=v_{1} u_{1} \cdots u_{s}$ is a longest path among these paths connecting $v_{1}$ and a pendant vertex in $G$. Let $G_{9}=G+v_{2} v_{3}+u_{s} v_{0}-v_{0} v_{3}-u_{0} v_{2}$, where $v_{0} \in\left(N\left(v_{3}\right) \cap V\left(C_{q}\right)\right) \backslash\left\{v_{1}\right\}$ and $u_{0} \in\left(N\left(v_{2}\right) \cap V\left(C_{q}\right)\right) \backslash\left\{v_{1}\right\}$ (Here, $u_{0}=v_{0}$ is also permitted).

If $s \geq 2$, then $M_{f}\left(G_{9}\right)=M_{f}(G)$ and hence $G_{9}$ is also an extremal graph of $\Gamma(\pi)$ such that $\left\{v_{1}, v_{2}, v_{3}\right\}$ forms a triangle in $G_{9}$, and hence Claim 4 holds. Otherwise, $s=1$. In this case, $M_{f}\left(G_{9}\right)-M_{f}(G)=$ $f\left(d\left(v_{1}\right), 2\right)+f(2,1)-f\left(d\left(v_{1}\right), 1\right)-f(2,2)$. By the definition of $f(x, y), G_{9}$ is also an extremal graph of $\Gamma(\pi)$ such that $\left\{v_{1}, v_{2}, v_{3}\right\}$ forms a triangle in $G_{9}$.

Lemma 2.6 Let $G$ be an extremal c-cyclic graph of $\Gamma(\pi)$ with $d_{n}=1$ and $c \geq 1$. If $\left\{v_{2}, v_{3}, \ldots, v_{k}\right\} \subseteq$ $N_{\mathcal{R}(G)}\left(v_{1}\right)$ and $G\left[\left\{v_{1}, v_{2}, v_{3}, \ldots, v_{k}\right\}\right] \subseteq \mathcal{R}(G)$, then there exists an extremal graph $G^{\prime} \in \Gamma(\pi)$ such that $G^{\prime}$ 
is a BFS-graph with $\left\{v_{2}, v_{3}, \ldots, v_{k}\right\} \subseteq N_{\mathcal{R}\left(G^{\prime}\right)}\left(v_{1}\right)$ and $G\left[\left\{v_{1}, v_{2}, v_{3}, \ldots, v_{k}\right\}\right] \subseteq \mathcal{R}\left(G^{\prime}\right)$.

Proof. We create an ordering $\prec$ of $V(G)$ by the breadth-first-search method as follows: Firstly, let $v_{1} \prec v_{2} \prec \ldots \prec v_{k}$; secondly, append all neighbors $u_{k+1}, u_{k+2}, \ldots, u_{d_{1}+1}$ of $N\left(v_{1}\right) \backslash\left\{v_{2}, v_{3}, \ldots, v_{k}\right\}$ to the ordered list, these neighbors are ordered such that $u \prec v$ whenever $d(u)>d(v)$ (in the remaining case the ordering can be arbitrary); thirdly append all neighbors of $N\left(v_{2}\right) \backslash N\left[v_{1}\right]$ to the ordered list, these neighbors are ordered such that $u \prec v$ whenever $d(u)>d(v)$ (in the remaining case the ordering can be arbitrary). Then, with the same method we can append the vertices $N\left(v_{i}\right) \backslash\left(N\left[v_{1}\right] \cup N\left(v_{2}\right) \cup \cdots \cup N\left(v_{i-1}\right)\right)$ in the ordered list, where $3 \leq i \leq k$, and then to the vertices $N(w) \backslash\left(N\left[v_{1}\right] \cup N\left(v_{2}\right) \cup \cdots \cup N\left(v_{k}\right)\right)$, where $d(w)=\max \left\{d(z): z \in N\left(v_{1}\right) \backslash\left\{v_{2}, v_{3}, \ldots, v_{k}\right\}\right\}$. Continue recursively with all vertices $v_{1}, v_{2}, \ldots, v_{n}$ until all vertices of $G$ are processed. It suffices to show the following two claims.

Claim 1. If $i<j$, then $d(u) \geq d(v)$ holds for any $u \in \mathcal{A}_{i}$ and $v \in \mathcal{A}_{j}$.

Suppose that there exist vertices $u$ and $v$ with $h(u)<h(v)$, while $d(u)<d(v)$. Furthermore, for convenience, we may choose $u$ as the first vertex in the ordering $\prec$ with such property and let $d(v)=$ $\max \{d(w)$ : where $h(w)>h(u)\}$. Then, $1 \leq i<j$ and $u \notin\left\{v_{1}, v_{2}, \ldots, v_{k}\right\}$.

If $u \in V\left(P_{v v_{1}}\right)$, then we choose $u^{\prime} \in \mathcal{A}_{i-1} \cap V\left(P_{v v_{1}}\right)$ such that $u^{\prime} u \in E(G)$. Since $d(v)>d(u)$, there exits vertex $v^{\prime} \in N(v) \backslash V\left(P_{v v_{1}}\right)$ such that $v^{\prime} \notin N(u)$. By the choice of $u$ and $v^{\prime}$, we have $d\left(u^{\prime}\right) \geq d\left(v^{\prime}\right)$ and $d(v)>d(u)$. Let $G_{1}=G+u v^{\prime}+u^{\prime} v-u^{\prime} u-v v^{\prime}$. By Lemma2.1 and the choice of $G$, we can conclude that $G_{1}$ is also an extremal graph of $\Gamma(\pi)$. Now, we construct a new ordering $\prec^{\prime}$ of $V\left(G_{1}\right)$ with the similar method as $\prec$. We suppose that $v_{1} \prec v_{2} \prec \cdots \prec v_{k} \prec u_{1} \prec u_{2} \prec \cdots \prec u_{t} \prec u$ are the first $t+k+1$ elements in the ordering $\prec$ of $V(G)$. By the choice of $u$ and $v$, we have $v_{1} \prec^{\prime} v_{2} \prec^{\prime} \cdots \prec^{\prime} v_{k} \prec^{\prime} u_{1} \prec^{\prime} u_{2} \prec^{\prime} \cdots \prec^{\prime} u_{t} \prec^{\prime} v$ are the first $t+k+1$ elements in the ordering $\prec^{\prime}$ of $V\left(G_{1}\right)$. By the choice of $u$ and $v$, for every $w \in\left\{v_{1}, v_{2}, \ldots, v_{k}, u_{1}, u_{2}, \ldots, u_{t}, v\right\}$, if $w \in \mathcal{A}_{t}\left(G_{1}\right)$, then $d_{G_{1}}(w) \geq d_{G_{1}}\left(w^{\prime}\right)$ holds for every $w^{\prime} \in \mathcal{A}_{s}\left(G_{1}\right)$, where $t<s$.

In what follows, we suppose that $u \notin V\left(P_{v v_{1}}\right)$.

Choose $w \in V\left(P_{v v_{1}}\right) \cap \mathcal{A}_{j-1}$ and choose $u^{\prime} \in \mathcal{A}_{i-1}$ such that $w v \in E(G)$ and $u^{\prime} u \in E(G)$. By the former argument, we may suppose that $u \neq w$.

If $u w \notin E(G)$, by the choice of $u$ and $j-1>i-1, d\left(u^{\prime}\right) \geq d(w)$. Recall that $d(v)>d(u)$. Let $G_{2}=G+u w+u^{\prime} v-u u^{\prime}-v w$. By Lemma 2.1, $G_{2}$ is also an extremal graph of $\Gamma(\pi)$.

Otherwise, $u w \in E(G)$. Recall that $d(v)>d(u)$. There exists vertex $w^{\prime} \in N(v)$ such that $w^{\prime} \notin V\left(P^{\prime}\right) \cup$ 
$N[u]$, where $P^{\prime}$ is a path connected $v$ and $u$ with $\left\{u^{\prime}, v_{1}, w\right\} \subseteq V\left(P^{\prime}\right)$. Let $G_{2}=G+u w^{\prime}+u^{\prime} v-u u^{\prime}-v w^{\prime}$. By the choice of $u$ and $j-1>i-1, d\left(u^{\prime}\right) \geq d\left(w^{\prime}\right)$. In this case, Lemma 2.1 implies that $G_{2}$ is also an extremal graph of $\Gamma(\pi)$.

Now, we construct a new ordering $\prec^{\prime}$ of $V\left(G_{2}\right)$ with the similar method as $\prec$. We suppose that $v_{1} \prec v_{2} \prec \cdots \prec v_{k} \prec u_{1} \prec u_{2} \prec \cdots \prec u_{t} \prec u$ are the first $t+k+1$ elements in the ordering $\prec$ of $V(G)$. By the choice of $u$ and $v$, we have $v_{1} \prec^{\prime} v_{2} \prec^{\prime} \ldots \prec^{\prime} v_{k} \prec^{\prime} u_{1} \prec^{\prime} u_{2} \prec^{\prime} \ldots \prec^{\prime} u_{t} \prec^{\prime} v$ are the first $t+k+1$ elements in the ordering $\prec^{\prime}$ of $V\left(G_{2}\right)$. By the choice of $u$ and $v$, for every $w \in\left\{v_{1}, v_{2}, \ldots, v_{k}, u_{1}, u_{2}, \ldots, u_{t}, v\right\}$, if $w \in \mathcal{A}_{t}\left(G_{2}\right)$, then $d_{G_{2}}(w) \geq d_{G_{2}}\left(w^{\prime}\right)$ holds for every $w^{\prime} \in \mathcal{A}_{s}\left(G_{2}\right)$, where $t<s$.

Repeating the above process finitely many times, we can achieve a graph $G^{*}$ and an ordering $\prec^{*}$ such that Claim 1 holds for $G^{*}$. Thus, we may directly suppose that Claim 1 holds for $G$.

Claim 2. If $h\left(u^{\prime}\right)=h\left(v^{\prime}\right)=i$ and $u^{\prime} \prec v^{\prime}$, then $d(u) \geq d(v)$ holds for every $u \in\left(N\left(u^{\prime}\right) \cap \mathcal{A}_{i+1}\right) \backslash N\left(v^{\prime}\right)$ and every $v \in\left(N\left(v^{\prime}\right) \cap \mathcal{A}_{i+1}\right) \backslash N\left(u^{\prime}\right)$.

If Claim 2 does not hold, we may suppose that $u^{\prime}$ is the first vertex in the ordering $\prec$ with the property that there exist vertices $v^{\prime} \in \mathcal{A}_{i}$ (suppose that $\left.u^{\prime} \in \mathcal{A}_{i}\right), u \in\left(N\left(u^{\prime}\right) \cap \mathcal{A}_{i+1}\right) \backslash N\left(v^{\prime}\right)$ and $v \in\left(N\left(v^{\prime}\right) \cap \mathcal{A}_{i+1}\right) \backslash N\left(u^{\prime}\right)$ such that $u^{\prime} \prec v^{\prime}$, but $d(u)<d(v)$. Furthermore, according to this, we may suppose that $u$ is the first vertex in the ordering $\prec$ and suppose that $d(v)=\max \left\{d(z): z \in \mathcal{A}_{i+1} \backslash N\left(u^{\prime}\right)\right.$, where $u \prec z\}$. It is easy to see that $u \notin\left\{v_{1}, v_{2}, \ldots, v_{k}\right\}$. Let $G_{3}=G+u^{\prime} v+v^{\prime} u-u^{\prime} u-v^{\prime} v$. Then, $G_{3} \in \Gamma(\pi)$. By the choice of $u$, we have $d\left(u^{\prime}\right) \geq d\left(v^{\prime}\right)$. Thus, Lemma 2.1 implies that $G_{3}$ is also an extremal graph of $\Gamma(\pi)$. Now, we construct a new ordering $\prec^{\prime}$ of $V\left(G_{3}\right)$ with the similar method as $\prec$. We suppose that $v_{1} \prec v_{2} \prec \cdots \prec v_{k} \prec u_{1} \prec u_{2} \prec \cdots \prec u_{t} \prec u$ are the first $t+k+1$ elements in the ordering $\prec$ of $V(G)$. By the choice of $u$ and $v, v_{1} \prec^{\prime} v_{2} \prec^{\prime} \ldots \prec^{\prime} v_{k} \prec^{\prime} u_{1} \prec^{\prime} u_{2} \prec^{\prime} \ldots \prec^{\prime} u_{t} \prec^{\prime} u$ are the first $t+k+1$ elements in the ordering $\prec^{\prime}$ of $V\left(G_{3}\right)$. By the choice of $u$ and $v$, for every $z \in\left\{v_{1}, v_{2}, \ldots, v_{k}, u_{1}, u_{2}, \ldots, u_{t}, v\right\} \cap \mathcal{A}_{i+1}\left(G_{3}\right)$, if $w \in \mathcal{A}_{i+1}\left(G_{3}\right)$ and $z \prec^{\prime} w$, then $d_{G_{3}}(z) \geq d_{G_{3}}(w)$

Repeating the above process finitely many times, we can obtain a graph $G^{* *}$ and an ordering $\prec^{* *}$ of $V\left(G^{* *}\right)$ such that $M_{f}\left(G^{* *}\right)=M_{f}(G)$ and Claim 2 holds for $G^{* *}$.

This completes the proof of this result.

Theorem 2.1 If $d_{n}=1$, then there exists an extremal graph $G \in \Gamma(\pi)$ such that $G$ is a BFS-graph. Furthermore, $\left\{v_{1}, v_{2}, v_{3}\right\}$ forms a triangle of $G$ when $c \geq 1$. 
Proof. We may suppose that $c \geq 1$, as the case of $c=0$ can be proved easily with the same argument. By Lemma 2.5. we may suppose that $G$ is an extremal graph in $\Gamma(\pi)$ such that $G$ contains a triangle with $V\left(C_{3}\right)=\left\{v_{1}, v_{2}, v_{3}\right\}$. Now, the result follows from Lemma 2.6.

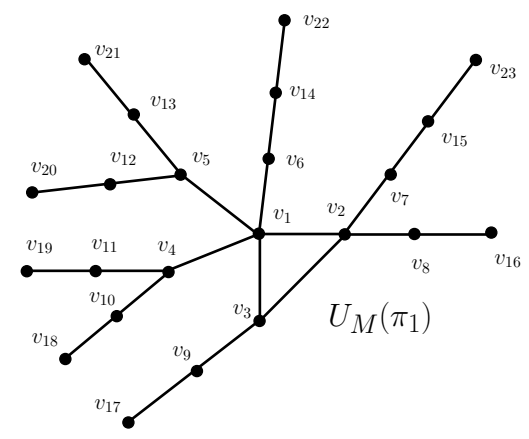

Figure 2.1: The unicyclic graph $U_{M}\left(\pi_{1}\right)$.

Hereafter, we use the symbol $p^{(q)}$ to define $q$ copies of the real number $p$. Suppose $\pi=\left(d_{1}, d_{2}, \ldots, d_{n}\right)$, where $d_{n}=1$. It has been shown that the $B F S$-tree is unique for any tree degree sequence $\pi$ [21]. Actually, we can construct a unique unicyclic $B F S$-graph $U_{M}(\pi)$ by the following breadth-first-search method for any unicyclic degree sequence $\pi$ : The unique cycle of $U_{M}(\pi)$ is a triangle with $V\left(C_{3}\right)=$ $\left\{v_{1}, v_{2}, v_{3}\right\}$. Select the vertex $v_{1}$ as the root vertex and begin with $v_{1}$ of the zeroth layer. Select the vertices $v_{2}, v_{3}, v_{4}, v_{5}, \ldots, v_{d_{1}+1}$ as the first layer such that $N\left(v_{1}\right)=\left\{v_{2}, v_{3}, v_{4}, v_{5}, \ldots, v_{d_{1}+1}\right\}$. Let $N\left(v_{2}\right)=$ $\left\{v_{1}, v_{3}, v_{d_{1}+2}, v_{d_{1}+3}, \ldots, v_{d_{1}+d_{2}-1}\right\}$ and $N\left(v_{3}\right)=\left\{v_{1}, v_{2}, v_{d_{1}+d_{2}}, \ldots, v_{d_{1}+d_{2}+d_{3}-3}\right\}$. Then, append $d_{4}-1$ vertices to $v_{4}$ such that $N\left(v_{4}\right)=\left\{v_{1}, v_{d_{1}+d_{2}+d_{3}-2}, \ldots, v_{d_{1}+d_{2}+d_{3}+d_{4}-4}\right\} \cdots$. Informally, for a given unicyclic degree sequence $\pi_{1}=\left(5,4,3^{(3)}, 2^{(10)}, 1^{(8)}\right), U_{M}\left(\pi_{1}\right)$ is the unicyclic graph as shown in Figure 2.1.

Corollary 2.1 Let $\pi$ be a c-cyclic degree sequence with $d_{n}=1$.

(i) [16] If $c=0$, then the BFS-tree is an extremal graph of $\Gamma(\pi)$.

(ii) 20] If $c=1$, then $U_{M}(\pi)$ is an extremal graph of $\Gamma(\pi)$.

Proof. One can easily check that $(i)$ and $(i i)$ follows from Theorem 2.1

Paths $P_{l_{1}}, P_{l_{2}}, \ldots, P_{l_{k}}$ are said to have almost equal lengths if $l_{1}, l_{2}, \ldots, l_{k}$ satisfy $\left|l_{i}-l_{j}\right| \leq 1$ for $1 \leq i \leq j \leq k$. In what follows, let $B_{1}, B_{2}, \ldots, B_{7}$ be seven bicyclic graphs as shown in Figure 2.2. If $\pi=\left(d_{1}, d_{2}, \ldots, d_{n}\right)$ is a bicyclic degree sequence, then $\sum_{i=1}^{n} d_{i}=2 n+2$, which implies that $\pi$ should be one of the following four cases. Moreover, we construct a special bicyclic graph $B_{M}(\pi)$ of $\Gamma(\pi)$ as follows: 
(i) If $d_{n}=1$ and $d_{1} \geq d_{2} \geq 3$, let $B_{M}(\pi)$ be a $B F S$-graph such that $\mathcal{R}\left(B_{M}(\pi)\right) \cong B_{1}$ and the remaining vertices appear in a $B F S$-ordering.

(ii) If $d_{1} \geq 5>d_{2}=2$ and $d_{n}=1$, let $B_{M}(\pi)$ be the bicyclic graph with $n$ vertices obtained from $B_{2}$ by attaching $d_{1}-4$ paths of almost equal lengths to the maximum degree vertex of $B_{2}$.

(iii) If $\pi=\left(4,2^{(n-1)}\right)$, let $B_{M}(\pi) \cong B_{3}$.

(iv) If $\pi=\left(3^{(2)}, 2^{(n-2)}\right)$, let $B_{M}(\pi) \cong B_{5}$.

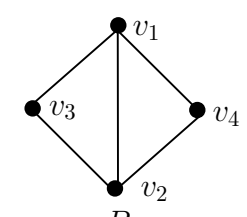

$B_{1}$

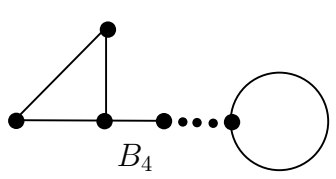

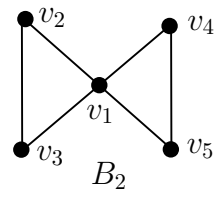

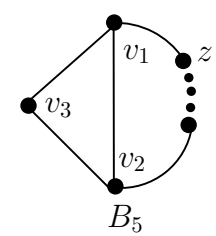

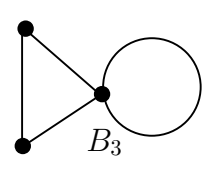

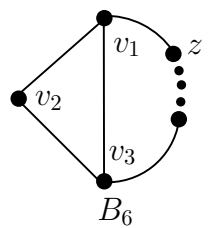

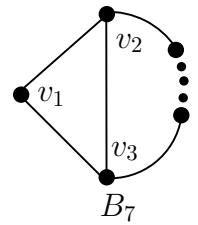

Figure 2.2: The bicyclic graphs $B_{1}, B_{2}, \ldots, B_{7}$.

Lemma 2.7 If $\pi$ is a bicyclic degree sequence with $d_{1} \geq d_{2} \geq 3$ and $d_{n}=1$, then $B_{M}(\pi)$ is an extremal bicyclic graph of $\Gamma(\pi)$.

Proof. By Theorem 2.1. we may suppose that $G$ is an extremal bicyclic graph of $\Gamma(\pi)$ such that $\left\{v_{1}, v_{2}, v_{3}\right\}$ forms a triangle $\Delta_{1}$ of $G$ and $G$ is an $B F S$-graph. We firstly prove the following two claims.

Claim 1. There is an extremal bicyclic graph $G$ of $\Gamma(\pi)$ such that $\left\{v_{1}, v_{2}, v_{3}\right\}$ forms a triangle $\Delta_{1}$ of $G$ and $G$ contains a cycle $C_{q}$ with $\left\{v_{1} v_{2}, v_{1} v_{4}\right\} \subseteq E\left(C_{q}\right)$.

Since $G$ is a bicyclic graph, there is another cycle, say $C_{q}$ such that $C_{q} \neq \Delta_{1}$. If $v_{1} v_{2}$ does not lie on $C_{q}$, then $\mathcal{R}(G) \cong B_{3}$ or $\mathcal{R}(G) \cong B_{4}$. It is easy to see that there exists at least one edge $u v$ on $C_{q}$ such that $d(u) \geq d(v),\left\{v_{1}, v_{2}, v_{3}\right\} \cap\{u, v\}=\varnothing$ and $d_{5} \geq d(v)$, by Lemma 2.4 and Remark 2.1, there exits another extremal graph $G^{\prime}$ such that $\Delta_{1}$ is also a triangles of $G^{\prime}, v_{1} v_{2}$ also lies on a cycle of $G^{\prime}$ and $\left\{v_{2}, v_{3}, v_{4}\right\} \subseteq N_{G^{\prime}}\left(v_{1}\right)$. Thus, we may suppose that $\Delta_{1}$ is a triangle of $G, v_{1} v_{2}$ lies on the cycle $C_{q}$ of $G$ and $\left\{v_{2}, v_{3}, v_{4}\right\} \subseteq N_{G}\left(v_{1}\right)$. In this case, $\mathcal{R}(G) \in\left\{B_{5}, B_{6}, B_{7}\right\}$. 
If either $v_{4} \in V\left(C_{q}\right)$ or $N\left(v_{4}\right) \cap\left\{v_{1}, v_{2}, v_{3}\right\} \neq\left\{v_{1}\right\}$, then Claim 1 already holds. Thus, we may suppose that $v_{4} \notin V\left(C_{q}\right)$ and $N\left(v_{4}\right) \cap\left\{v_{1}, v_{2}, v_{3}\right\}=\left\{v_{1}\right\}$ (In this case, $v_{4} \notin V(\mathcal{R}(G))$ ). Let $u_{0} \in N\left(v_{4}\right) \backslash\left\{v_{1}, v_{2}, v_{3}\right\}$ (such vertex exists since $G$ contains a cycle $C_{q}$ ).

If $\mathcal{R}(G) \cong B_{5}$ or $\mathcal{R}(G) \cong B_{7}$, we choose $u \in N\left(v_{2}\right) \cap V\left(B_{5}\right)$ such that $u \notin\left\{v_{1}, v_{3}\right\}$. Let $G_{1}=$ $G+u u_{0}+v_{2} v_{4}-v_{2} u-u_{0} v_{4}$. Since $d\left(v_{2}\right) \geq d\left(u_{0}\right)$ and $d\left(v_{4}\right) \geq d(u)$, by Lemma 2.1, $G_{1}$ is also an extremal graph of $\Gamma(\pi)$ such that Claim 1 holds.

If $\mathcal{R}(G) \cong B_{6}$, we choose $u \in N\left(v_{3}\right) \cap V\left(B_{6}\right)$ such that $u \notin\left\{v_{1}, v_{2}\right\}$. Let $G_{2}=G+u u_{0}+v_{3} v_{4}-v_{3} u-$ $v_{4} u_{0}$. Since $d\left(v_{3}\right) \geq d\left(u_{0}\right)$ and $d\left(v_{4}\right) \geq d(u)$, by Lemma 2.1. $G_{2}$ is also an extremal graph of $\Gamma(\pi)$ such that Claim 1 holds. This completes the proof of Claim 1.

Claim 2. There is an extremal bicyclic graph $G$ of $\Gamma(\pi)$ such that $\left\{v_{1}, v_{2}, v_{4}\right\}$ and $\left\{v_{1}, v_{2}, v_{3}\right\}$ form two triangles of $G$.

By Claim 1, we may suppose that $G$ is an extremal graph of $\Gamma(\pi)$ such that $\left\{v_{1}, v_{2}, v_{3}\right\}$ form a triangle of $G$ and $G$ contains a cycle $C_{q}$ with $\left\{v_{1} v_{2}, v_{1} v_{4}\right\} \subseteq E\left(C_{q}\right)$. In this case, either $\mathcal{R}(G) \cong B_{5}$ or $\mathcal{R}(G) \cong B_{6}$ with $z=v_{4}$.

If $v_{3} v_{4} \in E(G)$, then $\left\{v_{1}, v_{2}, v_{3}, v_{4}\right\}$ form a $C_{4}$ of $G$ (In this case, $\left.\mathcal{R}(G) \cong B_{6}\right)$. In this case, there exists some vertex $u \in N\left(v_{2}\right) \backslash V(\mathcal{R}(G))$ and $v_{2} v_{4} \notin E(G)$. Let $G_{3}=G+v_{2} v_{4}+v_{3} u-v_{3} v_{4}-v_{2} u$. By Lemma 2.1. $G_{3}$ is also an extremal graph of $\Gamma(\pi)$ such that Claim 2 holds.

Otherwise, $v_{3} v_{4} \notin E(G)$.

If $\mathcal{R}(G) \cong B_{6}$, then there exists a vertex $w_{2} \in N\left(v_{2}\right) \backslash V(\mathcal{R}(G))$. Let $G_{4}=G+v_{2} v_{4}+w_{2} w_{0}-v_{2} w_{2}-$ $v_{4} w_{0}$, where $w_{0} \in\left(N\left(v_{4}\right) \cap V\left(B_{6}\right)\right) \backslash\left\{v_{1}\right\}$. By Lemma 2.1. $G_{4}$ is an extremal graph of $\Gamma(\pi)$ such that Claim 2 holds.

Therefore, we may suppose that $\mathcal{R}(G) \cong B_{5}$ in what follows. Furthermore, we may suppose that $v_{2} v_{4} \notin E(G)$. Otherwise, Claim 1 already holds. We choose $u_{0} \in\left(N\left(v_{4}\right) \cap V\left(B_{5}\right)\right) \backslash\left\{v_{1}\right\}$ and $z_{0} \in$ $\left(N\left(v_{2}\right) \cap V\left(B_{5}\right)\right) \backslash\left\{v_{1}, v_{3}\right\}$, where $u_{0}=z_{0}$ is permitted.

If $d_{3} \geq 3$, then we choose $w \in N\left(v_{3}\right) \backslash V\left(B_{5}\right)$. Note that $w u_{0} \notin E(G)$. Let $G_{5}=v_{3} v_{4}+w u_{0}-v_{3} w-v_{4} u_{0}$. By Lemma 2.1, $G_{5}$ is an extremal graph of $\Gamma(\pi)$ such that $\left\{v_{1}, v_{2}, v_{3}, v_{4}\right\}$ form a $C_{4}$ and $\left\{v_{1}, v_{2}, v_{3}\right\}$ form a triangle of $G_{5}$. From the above argument, Claim 2 holds (see the case of $v_{3} \in N\left(v_{4}\right)$ and $\mathcal{R}(G) \cong B_{6}$ ).

Otherwise, $d_{3}=2$, and hence $d_{1} \geq 4\left(\right.$ since $d_{n}=1$ and $\left.\sum_{i=1}^{n} d_{i}=2(n+1)\right)$. Let $w_{1}$ be a vertex of $N\left(v_{1}\right) \backslash\left\{v_{2}, v_{3}, v_{4}\right\}$, and let $G_{6}=G+v_{2} v_{4}+z_{0} v_{1}+w_{1} u_{0}-v_{2} z_{0}-v_{4} u_{0}-v_{1} w_{1}$. Now, by 1.2 it follows 
that $M_{f}\left(G_{6}\right)-M_{f}(G)=f\left(d_{1}, 2\right)+f\left(2, d\left(w_{1}\right)\right)-f\left(d_{1}, d\left(w_{1}\right)\right)-f(2,2)$, and hence $G_{6}$ is also an extremal graph of $\Gamma(\pi)$ such that Claim 2 holds.

Now, by Claim 2 and Lemma 2.6 we have $G \cong B_{M}(\pi)$, as desired.

Theorem 2.2 If $\pi$ is a bicyclic degree sequence, then $B_{M}(\pi)$ is an extremal graph of $\Gamma(\pi)$.

Proof. Since $\pi=\left(d_{1}, d_{2}, \ldots, d_{n}\right)$ is a bicyclic degree sequence, then $\sum_{i=1}^{n} d_{i}=2 n+2$, and hence it suffices to consider the following four cases.

If $\pi=\left(4,2^{(n-1)}\right)$ (respectively, $\left(3^{(2)}, 2^{(n-2)}\right)$ ), it is easy to check that $B_{3}$ (respectively, $B_{5}$ ) is an extremal graph of $\Gamma(\pi)$. If $d_{1} \geq d_{2} \geq 3$ and $d_{n}=1$, then the result follows from Lemma 2.7

Otherwise, $d_{1} \geq 5>d_{2}=2$ and $d_{n}=1$. In this case, by Theorem 2.1. we may suppose that $G$ is an extremal bicyclic graph of $\Gamma(\pi)$ such that $G$ is a $B F S$-graph obtained by attaching $d_{1}-4$ paths of almost equal lengths to the maximum degree vertex of $B_{3}$. Now, we assume that $B_{3} \neq B_{2}$, and hence $B_{3}$ contains a cycle $C_{q}$, where $q \geq 4$. We suppose that $N\left(v_{1}\right) \cap V\left(C_{q}\right)=\left\{u_{1}, w_{1}\right\}, N\left(u_{1}\right) \cap V\left(C_{q}\right)=\left\{v_{1}, u_{2}\right\}$ and $N\left(w_{1}\right) \cap V\left(C_{q}\right)=\left\{v_{1}, w_{2}\right\}$, where $w_{2}=u_{2}$ is permitted. We choose $z$ as a pendant vertex such that the distance between $v_{1}$ and $z$ is as small as possible, and suppose that $z_{1} \in N(z)$.

Let $G_{1}=G+u_{1} w_{1}+z w_{2}-u_{1} u_{2}-w_{1} w_{2}$.

If $z_{1}=v_{1}$, then $M_{f}\left(G_{1}\right)-M_{f}(G)=f\left(d\left(v_{1}\right), 2\right)+f(2,1)-f\left(d\left(v_{1}\right), 1\right)-f(2,2)$. If $z_{1} \neq v_{1}$, then $M_{f}\left(G_{1}\right)=M_{f}(G)$. In both case, $G_{1}$ is also an extremal bicyclic graph of $\Gamma(\pi)$ such that $\mathcal{R}\left(G_{1}\right) \cong B_{2}$. Now, the result follows from Lemma 2.6 .

\section{The majorization theorem of connectivity function}

If $\pi=\left(d_{1}, d_{2}, \ldots, d_{n}\right)$ is a non-increasing integer sequence and $d_{i} \geq d_{j}+2$ holds for some integers $1 \leq i<j \leq n$, then the following operation is called a unit transformation from $i$ to $j$ on $\pi$ : subtract 1 from $d_{i}$ and add 1 to $d_{j}$. The following famous lemma about majorization for integer sequences is due to Muirhead (for instance, see [10,11]).

Lemma 3.1 (Muirhead Lemma) If $\pi$ and $\pi^{\prime}$ are two non-increasing integer sequences and $\pi \triangleleft \pi^{\prime}$, then $\pi$ can be obtained from $\pi^{\prime}$ by a finite sequence of unit transformations.

Lemma 3.2 Let $u, v$ be two vertices of a connected graph $G$ with $d(u) \geq d(v) \geq 2$ and $w$ be some vertex of $N(v) \backslash\left(V\left(P_{u v}\right) \cup N[u]\right)$. Let $G^{\prime}=G+w u-w v$. Suppose that $N(v) \backslash\{w, u\}=\left\{z_{1}^{\prime}, z_{2}^{\prime}, \ldots, z_{|N(v) \backslash\{w, u\}|}^{\prime}\right\}$, 
and there exist $\left\{z_{1}, z_{2}, \ldots, z_{|N(v) \backslash\{w, u\}|}\right\} \subseteq N(u) \backslash\{v\}$ such that $d\left(z_{i}\right) \geq d\left(z_{i}^{\prime}\right)$ for $1 \leq i \leq|N(v) \backslash\{w, u\}|$. If $f(x, y)$ is a good escalating function, then $M_{f}\left(G^{\prime}\right)>M_{f}(G)$.

Proof. If $u v \notin E(G)$, by 1.2 it follows that

$$
\begin{aligned}
& M_{f}\left(G^{\prime}\right)-M_{f}(G) \\
= & \sum_{z \in N(u)}(f(d(u)+1, d(z))-f(d(u), d(z)))-\sum_{z^{\prime} \in N(v) \backslash\{w\}}\left(f\left(d(v), d\left(z^{\prime}\right)\right)-f\left(d(v)-1, d\left(z^{\prime}\right)\right)\right) \\
+ & f(d(u)+1, d(w))-f(d(v), d(w)) .
\end{aligned}
$$

Let $g(x, y)=\frac{\partial f(x, y)}{\partial x}$. We may suppose that $N(v) \backslash\{u, w\}=\left\{z_{1}^{\prime}, z_{2}^{\prime}, \ldots, z_{d(v)-1}^{\prime}\right\}$. Then, there exists $\left\{z_{1}, z_{2}, \ldots, z_{d(v)-1}\right\} \subseteq N(u) \backslash\{v\}$ such that $d\left(z_{i}\right) \geq d\left(z_{i}^{\prime}\right)$. Recall that $d(u) \geq d(v), d\left(z_{i}\right) \geq d\left(z_{i}^{\prime}\right)$ and $\frac{\partial^{2} f(x, y)}{\partial x^{2}} \geq 0$. Thus, for $1 \leq i \leq d(v)-1$, there exists $0 \leq a \leq 1$ and $0 \leq b \leq 1$ such that

$$
\begin{aligned}
& f\left(d(u)+1, d\left(z_{i}\right)\right)-f\left(d(u), d\left(z_{i}\right)\right)-\left(f\left(d(v), d\left(z_{i}^{\prime}\right)\right)-f\left(d(v)-1, d\left(z_{i}^{\prime}\right)\right)\right) \\
= & f\left(d(u)+1, d\left(z_{i}\right)\right)-f\left(d(u), d\left(z_{i}\right)\right)-\left(f\left(d(v), d\left(z_{i}\right)\right)-f\left(d(v)-1, d\left(z_{i}\right)\right)\right) \\
& +f\left(d(v), d\left(z_{i}\right)\right)-f\left(d(v)-1, d\left(z_{i}\right)\right)-\left(f\left(d(v), d\left(z_{i}^{\prime}\right)\right)-f\left(d(v)-1, d\left(z_{i}^{\prime}\right)\right)\right) \\
\geq & f\left(d(u)+1, d\left(z_{i}\right)\right)-f\left(d(u), d\left(z_{i}\right)\right)-\left(f\left(d(v), d\left(z_{i}\right)\right)-f\left(d(v)-1, d\left(z_{i}\right)\right)\right) \\
= & g\left(d(u)+a, d\left(z_{i}\right)\right)-g\left(d(v)-1+b, d\left(z_{i}\right)\right) \geq 0,
\end{aligned}
$$

which implies that $M_{f}\left(G^{\prime}\right)-M_{f}(G) \geq f(d(u)+1, d(w))-f(d(v), d(w))>0$, as $g(x, y)>0$.

Otherwise, $u v \in E(G)$. In this case, by $(1.2)$ it follows that

$$
\begin{aligned}
& M_{f}\left(G^{\prime}\right)-M_{f}(G) \\
= & \sum_{z \in N(u) \backslash\{v\}}(f(d(u)+1, d(z))-f(d(u), d(z)))-\sum_{z^{\prime} \in N(v) \backslash\{u, w\}}\left(f\left(d(v), d\left(z^{\prime}\right)\right)-f\left(d(v)-1, d\left(z^{\prime}\right)\right)\right) \\
& +f(d(u)+1, d(w))-f(d(v), d(w))+f(d(u)+1, d(v)-1)-f(d(u), d(v)) .
\end{aligned}
$$

Since $|N(u) \backslash\{v\}|>|N(v) \backslash\{u, w\}|$, there exists some vertex $z_{0} \in N(u) \backslash\{v\}$ such that

$$
\begin{aligned}
& \sum_{z \in N(u) \backslash\{v\}}(f(d(u)+1, d(z))-f(d(u), d(z)))-\sum_{z^{\prime} \in N(v) \backslash\{u, w\}}\left(f\left(d(v), d\left(z^{\prime}\right)\right)-f\left(d(v)-1, d\left(z^{\prime}\right)\right)\right) \\
& \geq f\left(d(u)+1, d\left(z_{0}\right)\right)-f\left(d(u), d\left(z_{0}\right)\right)>0 .
\end{aligned}
$$


Furthermore, since $f(x, y)$ is good escalating, we can conclude that

$$
M_{f}\left(G^{\prime}\right)-M_{f}(G)>f(d(u)+1, d(w))-f(d(v), d(w))+f(d(u)+1, d(v)-1)-f(d(u), d(v))
$$

$\geq 0$.

This completes the proof of this result.

Suppose that $\pi=\left(d_{1}, d_{2}, \ldots, d_{n}\right)$ and $\pi^{\prime}=\left(d_{1}^{\prime}, d_{2}^{\prime}, \ldots, d_{n}^{\prime}\right)$ are two $c$-cyclic graphic degree sequences. If $\pi \triangleleft \pi^{\prime}$, by Lemma 3.1, we may suppose that $\pi$ and $\pi^{\prime}$ differ only in two positions where the difference is 1 , that is, $d_{i}=d_{i}^{\prime}, i \neq p, q, 1 \leq p<q \leq n$, and $d_{p}^{\prime}=d_{p}+1, d_{q}^{\prime}=d_{q}-1$.

Let $G$ and $G^{\prime}$ be an extremal $c$-cyclic graph of $\Gamma(\pi)$ and $\Gamma\left(\pi^{\prime}\right)$, respectively. If $w$ is a vertex of $G$ such that $w \in N\left(v_{q}\right) \backslash\left(V\left(P_{v_{p} v_{q}}\right) \cup N\left[v_{p}\right]\right)$, and there exists $\left\{z_{1}, z_{2}, \ldots, z_{\left|N\left(v_{q}\right) \backslash\left\{w, v_{p}\right\}\right|}\right\} \subseteq N\left(v_{p}\right) \backslash\left\{v_{q}\right\}$ such that $d\left(z_{i}\right) \geq d\left(z_{i}^{\prime}\right)$ for $1 \leq i \leq\left|N\left(v_{q}\right) \backslash\left\{w, v_{p}\right\}\right|$, where $N\left(v_{q}\right) \backslash\left\{w, v_{p}\right\}=\left\{z_{1}^{\prime}, z_{2}^{\prime}, \ldots, z_{\left|N\left(v_{q}\right) \backslash\left\{w, v_{p}\right\}\right|}^{\prime}\right.$, then we call $w$ a surprising vertex of $G$. If $f(x, y)$ is good escalating and $G$ contains some surprising vertex $w$, then let $G_{1}=G+v_{p} w-v_{q} w$. Thus, $G_{1} \in \Gamma\left(\pi^{\prime}\right)$. Since $p<q, d\left(v_{p}\right) \geq d\left(v_{q}\right)$ follows from Theorem 2.1. By Lemma 3.2. we have $M_{f}(G)<M_{f}\left(G_{1}\right) \leq M_{f}\left(G^{\prime}\right)$. Thus,

if $G$ contains a surprising vertex, then $M_{f}(G)<M_{f}\left(G^{\prime}\right)$.

Zhang et al. 22] showed that the size of $M_{f}(T)$ and $M_{f}\left(T^{\prime}\right)$ can be deduced from the relation $\pi \triangleleft \pi^{\prime}$ when $f(x, y)$ is a special symmetric bivariable function for any extremal trees $T$ and $T^{\prime}$ of $\Gamma(\pi)$ and $\Gamma\left(\pi^{\prime}\right)$, respectively. Actually, if $\pi \triangleleft \pi^{\prime}$ and $f(x, y)$ is good escalating, by Corollary 2.1 (i) we can conclude that the BFS-tree of $\Gamma(\pi)$ contains a surprising vertex. Thus, by (3.1) and Corollary 2.1 ( $i$ ) it follows that

Theorem 3.1 Let $\pi$ and $\pi^{\prime}$ be two different non-increasing tree degree sequences with $\pi \triangleleft \pi^{\prime}$. Let $T$ and $T^{\prime}$ be an extremal tree of $\Gamma(\pi)$ and $\Gamma\left(\pi^{\prime}\right)$, respectively. If $f(x, y)$ is a good escalating function, then $M_{f}(T)<M_{f}\left(T^{\prime}\right)$.

Theorem 3.2 Let $\pi$ and $\pi^{\prime}$ be two different non-increasing unicyclic degree sequences with $\pi \triangleleft \pi^{\prime}$. Let $U$ and $U^{\prime}$ be an extremal unicyclic graph of $\Gamma(\pi)$ and $\Gamma\left(\pi^{\prime}\right)$, respectively. If $f(x, y)$ is a good escalating function, then $M_{f}(U)<M_{f}\left(U^{\prime}\right)$.

Proof. Suppose that $\pi=\left(d_{1}, d_{2}, \ldots, d_{n}\right)$ and $\pi^{\prime}=\left(d_{1}^{\prime}, d_{2}^{\prime}, \ldots, d_{n}^{\prime}\right)$. Since $\pi \triangleleft \pi^{\prime}$, by Lemma 3.1 we may suppose that $\pi$ and $\pi^{\prime}$ differ only in two positions, where the differences are 1 . Thus, we may assume that $d_{i}=d_{i}^{\prime}$ for $i \neq p, q$, and $d_{p}+1=d_{p}^{\prime}, d_{q}-1=d_{q}^{\prime}$. 
If $d_{n}=2$, then $\pi=(2,2, \ldots, 2)$ and $\pi^{\prime}=(3,2, \ldots, 2,1)$. By Corollary 2.1 (ii), it suffices to show that $M_{f}\left(C_{n}\right)<M_{f}\left(U_{M}\left(\pi^{\prime}\right)\right)$. Since $U_{M}\left(\pi^{\prime}\right)$ contains at least one pendant vertex, $n \geq 4$. We may suppose that $n \geq 5$, as the case of $n=4$ can be proved similarly. Let $g(x, y)=\frac{\partial f(x, y)}{\partial x}$. In this case, since $\frac{\partial^{2} f(x, y)}{\partial x^{2}} \geq 0$ and $g(x, y)>0$, by Corollary 2.1 (ii) we have

$$
\begin{aligned}
M_{f}\left(U_{M}\left(\pi^{\prime}\right)\right)-M_{f}\left(C_{n}\right) & =3 f(3,2)+f(2,1)-4 f(2,2) \\
& >f(3,2)+f(2,1)-2 f(2,2) \\
& =f(3,2)-f(2,2)-(f(2,2)-f(1,2)) \\
& =g(2+a, 2)-g(1+b, 2) \geq 0,
\end{aligned}
$$

where $0 \leq a \leq 1$ and $0 \leq b \leq 1$.

Otherwise, $d_{n}=1$. In this case, it suffices to show that $M_{f}\left(U_{M}(\pi)\right)<M_{f}\left(U_{M}\left(\pi^{\prime}\right)\right)$ by Corollary 2.1 (ii). If $2 \leq q \leq 3$, then $d_{q} \geq 3$ since $U_{M}\left(\pi^{\prime}\right) \in \Gamma\left(\pi^{\prime}\right)$. If $q \geq 4$, then $d_{q} \geq 2$. In both cases, $U_{M}(\pi)$ contains a surprising vertex. Now, the result follows from (3.1).

Theorem 3.3 Let $\pi$ and $\pi^{\prime}$ be two different non-increasing bicyclic degree sequences with $\pi \triangleleft \pi^{\prime}$. Let $B$ and $B^{\prime}$ be an extremal bicyclic graph of $\Gamma(\pi)$ and $\Gamma\left(\pi^{\prime}\right)$, respectively. If $f(x, y)$ is a good escalating function, then $M_{f}(B)<M_{f}\left(B^{\prime}\right)$.

Proof. Suppose that $\pi=\left(d_{1}, d_{2}, \ldots, d_{n}\right)$ and $\pi^{\prime}=\left(d_{1}^{\prime}, d_{2}^{\prime}, \ldots, d_{n}^{\prime}\right)$. Since $\pi \triangleleft \pi^{\prime}$, by Lemma 3.1 we may suppose that $\pi$ and $\pi^{\prime}$ differ only in two positions, where the differences are 1 . Thus, we may assume that $d_{i}=d_{i}^{\prime}$ for $i \neq p, q$, and $d_{p}+1=d_{p}^{\prime}, d_{q}-1=d_{q}^{\prime}$. It suffices to show that $M_{f}\left(B_{M}(\pi)\right)<M_{f}\left(B_{M}\left(\pi^{\prime}\right)\right)$ by Theorem 2.2 .

We firstly consider the case of $d_{n} \geq 2$. In this case, either $\pi=\left(4,2^{(n-1)}\right)$ or $\pi=\left(3^{(2)}, 2^{(n-2)}\right)$. If $\pi=\left(4,2^{(n-1)}\right)$, then either $\pi^{\prime}=\left(5,2^{(n-2)}, 1\right)$ or $\pi^{\prime}=\left(4,3,2^{(n-3)}, 1\right)$. If $\pi=\left(3^{(2)}, 2^{(n-2)}\right)$, then $\pi^{\prime}=\left(4,2^{(n-1)}\right)$ or $\pi^{\prime}=\left(3^{(3)}, 2^{(n-4)}, 1\right)$ or $\pi^{\prime}=\left(4,3,2^{(n-3)}, 1\right)$. Whenever which case happens, according to Theorem 2.2, $B_{M}(\pi)$ contains a surprising vertex, and hence the result follows from 3.1. We secondly consider the case of $d_{n}=1$.

Case 1. $d_{n}=1$ and $d_{2}=2$.

In this case, $\pi=\left(k+4,2^{(n-k-1)}, 1^{(k)}\right)$, where $k \geq 1$. Then, either $\pi^{\prime}=\left(k+5,2^{(n-k-2)}, 1^{(k+1)}\right)$ or $\pi^{\prime}=\left(k+4,3,2^{(n-k-3)}, 1^{(k+1)}\right)$. According to Theorem $2.2, \mathcal{R}\left(B_{M}(\pi)\right) \cong B_{2}$. 
If $\pi^{\prime}=\left(k+4,3,2^{(n-k-3)}, 1^{(k+1)}\right)$, since $v_{5} \in N_{B_{M}(\pi)}\left(v_{4}\right) \backslash N_{B_{M}(\pi)}\left[v_{2}\right]$ and $v_{5} \notin V\left(P_{v_{2} v_{4}}\right)$, it is easy to see that $v_{5}$ is a surprising vertex of $B_{M}(\pi)$.

If $\pi^{\prime}=\left(k+5,2^{(n-k-2)}, 1^{(k+1)}\right)$, then $p=1, q \geq 6$ and $d_{q}=2$. By the structure of $B_{M}(\pi)$, there exists a vertex $v_{k}(k>q)$ such that $v_{k} \in N_{B_{M}(\pi)}\left(v_{q}\right) \backslash N_{B_{M}(\pi)}\left[v_{p}\right]$ and $v_{k} \notin V\left(P_{v_{p} v_{q}}\right)$. In this case, it is easy to see that $v_{k}$ is a surprising vertex of $B_{M}(\pi)$.

In both cases, the result follows from (3.1).

Case 2. $d_{n}=1$ and $d_{1} \geq d_{2} \geq 3$.

By Theorem 2.2. $\mathcal{R}\left(B_{M}(\pi)\right) \cong B_{1}$. If $q \geq 5$, then $d_{q}=d_{q}^{\prime}+1 \geq 2$. If $3 \leq q \leq 4$, since $B_{M}\left(\pi^{\prime}\right)$ is a bicyclic graph, $d_{q}=d_{q}^{\prime}+1 \geq 3$. In both cases, by the structure of $B_{M}(\pi)$, there exists vertex $v_{k}(k>q)$ such that $v_{k} \in N_{B_{M}(\pi)}\left(v_{q}\right) \backslash N_{B_{M}(\pi)}\left[v_{p}\right]$ and $v_{k} \notin V\left(P_{v_{p} v_{q}}\right)$. In this case, it is easy to see that $v_{k}$ is a surprising vertex of $B_{M}(\pi)$. Now, the result follows from (3.1). Thus, we may suppose that $q=2$, and hence $p=1$ in the sequel.

If $q=2$ and $d_{2} \geq 4$, since $\mathcal{R}\left(B_{M}(\pi)\right) \cong B_{1}$, there exists a vertex $v_{k} \in N_{B_{M}(\pi)}\left(v_{2}\right) \backslash N_{B_{M}(\pi)}\left[v_{1}\right]$ and $v_{k} \notin V\left(P_{v_{1} v_{2}}\right)$, which implies that $v_{k}$ is a surprising vertex of $B_{M}(\pi)$. Now, the result follows from 3.1.

If $q=2$ and $d_{2}=3$, then $d_{2}^{\prime}=2$. Thus, $\pi=\left(k+3,3,2^{(n-k-2)}, 1^{(k)}\right)$ and $\pi^{\prime}=\left(k+4,2^{(n-k-1)}, 1^{(k)}\right)$, where $k \geq 1$. By Theorem 2.2 , $\mathcal{R}\left(B_{M}(\pi)\right) \cong B_{1}$ and $\mathcal{R}\left(B_{M}\left(\pi^{\prime}\right)\right) \cong B_{2}$.

Then, $B_{M}(\pi)$ is obtained from $B_{1}$ by attaching $k$ paths, say $P_{l_{1}}, P_{l_{2}}, \ldots, P_{l_{k}}$, of almost equal lengths to one vertex of degree three of $B_{1}$, where $l_{1} \geq l_{2} \geq \cdots \geq l_{k} \geq 1$ and $l_{1} \geq 2$.

If $l_{1} \geq 3$, since $g(x, y)=\frac{\partial f(x, y)}{\partial x}>0$ and $\frac{\partial^{2} f(x, y)}{\partial x^{2}} \geq 0$, we have $M_{f}\left(B_{M}\left(\pi^{\prime}\right)\right)-M_{f}\left(B_{M}(\pi)\right)=$ $(k+4) f(k+4,2)+f(2,2)-(k+2) f(k+3,2)-f(k+3,3)-2 f(3,2)=(k+2) g(k+3+a, 2)-2 f(3,2)+$ $2 f(k+4,2)+f(2,2)-f(k+3,3)=(k+2) g(k+3+a, 2)-g(2+b, 2)+2 f(k+4,2)-f(k+3,3)-f(3,2)>$ $2 f(k+4,2)-f(k+3,3)-f(3,2) \geq 0$, where $0 \leq a \leq 1$ and $0 \leq b \leq 1$.

Otherwise, $l_{1}=2$. We may suppose that $\left(l_{1}, l_{2}, \ldots, l_{k}\right)=\left(2^{(s)}, 1^{(k-s)}\right)$, where $1 \leq s \leq k$. In this case, $M_{f}\left(B_{M}\left(\pi^{\prime}\right)\right)-M_{f}\left(B_{M}(\pi)\right)=(s+3) f(k+4,2)+2 f(2,2)+(k+1-s) f(k+4,1)-(s+2) f(k+3,2)-f(k+$ $3,3)-2 f(3,2)-(k-s) f(k+3,1)-f(2,1)>(s+2) g(k+3+a, 2)-2 g(2+b, 2)+f(k+4,2)+f(k+4,1)-f(k+$ $3,3)-f(2,1)>f(k+4,2)+f(k+4,1)-f(k+3,3)-f(2,1) \geq f(k+3,3)+f(3,1)-f(k+3,3)-f(2,1)>0$, where $0 \leq a \leq 1$ and $0 \leq b \leq 1$.

Let $F_{n}(k)$ (respectively, $F_{n}^{\prime}(k), F_{n}^{\prime \prime}(k)$ ) be the tree (respectively, unicyclic graph, bicyclic graph) on $n$ vertices obtained by attaching $k$ paths of almost equal lengths to one isolated vertex (respectively, one 
vertex of $C_{3}$, the vertex of degree four of $B_{2}$ ). From Theorems 2.1 2.2 and Theorems 3.1 3.3, we can deduce the following extremal results easily:

Corollary 3.1 If $k \geq 1$ and $f(x, y)$ is good escalating, then $F_{n}^{\prime \prime}(k)$ (respectively, $\left.F_{n}^{\prime}(k), F_{n}(k)\right)$ is an extremal bicyclic graph (respectively, unicyclic graph, tree) among all bicyclic graphs (respectively, unicyclic graphs, trees) with $n$ vertices and $k$ pendant vertices when $n \geq k+5$ (respectively, $n \geq k+3, n \geq k+1)$.

Proof. Here, we only prove the case of bicyclic graphs, as the cases of unicyclic graphs and trees can be proved similarly. Let $\pi^{\prime}=\left(k+4,2^{(n-k-1)}, 1^{(k)}\right)$. Suppose that $G$ is a bicyclic graph with $\pi=\left(d_{1}, d_{2}, \ldots, d_{n}\right)$ as its degree sequence, where $d_{n}=d_{n-1}=\cdots=d_{n-k+1}=1$ and $d_{n-k} \geq 2$. If $\pi \neq \pi^{\prime}$, then $\pi \triangleleft \pi^{\prime}$. Now, the result follows from Theorem 2.2 and Theorem 3.3 .

Let $S_{n}^{(0)}$ be the star with $n$ vertices, let $S_{n}^{(1)}$ be the unicyclic graph obtained from the star $S_{n}^{(0)}$ by adding one edge between two pendant vertices of $S_{n}^{(0)}$, and let $S_{n}^{(2)}$ be the bicyclic graph obtained from $B_{1}$ by attaching $n-4$ pendant vertices to one vertex of degree three of $B_{1}$.

Corollary 3.2 If $n \geq 4$ and $f(x, y)$ is good escalating, then $S_{n}^{(c)}$ is the unique extremal c-cyclic graph among all c-cyclic graphs with $n$ vertices for $0 \leq c \leq 2$.

Proof. Here, we only prove the case of bicyclic graphs, as the cases of unicyclic graphs and trees can be proved similarly. Let $\pi^{\prime}=\left(n-1,3,2^{(2)}, 1^{(n-4)}\right)$. Then, $\pi^{\prime}$ uniquely maximizes those degree sequences of bicyclic graphs with $n$ vertices in the relation $\triangleleft$. Furthermore, $S_{n}^{(2)}$ is the unique bicyclic graphs of $\Gamma\left(\pi^{\prime}\right)$. Now, the result follows from Theorem 3.3 .

\section{Some applications}

As an extension of $Z_{2}(G)$, we define $Z_{\alpha}(G)$ as follows:

$$
Z_{\alpha}(G)=\sum_{u v \in E(G)}(d(u)+d(v)-2)^{\alpha} .
$$

In [22, Zhang et al. has shown that $\chi_{\alpha}(G)$ is escalating for $\alpha \geq 1$ and $\chi_{\alpha}(G)$ is de-escalating for $0<\alpha<1$, and $W_{\alpha}(G)$ is escalating for $\alpha>0$. In the following, we will extend these results.

Theorem 4.1 Let $G$ be a connected graph with at least three vertices. Then, $(i) Z_{\alpha}(G)$ and $\chi_{\alpha}(G)$ are escalating for $\alpha>1$ and $\alpha<0$. Furthermore, $Z_{\alpha}(G)$ and $\chi_{\alpha}(G)$ are good escalating for $\alpha>1$. (ii) $Z_{\alpha}(G)$ and $\chi_{\alpha}(G)$ are de-escalating for $0<\alpha<1$. 
Proof. Here, we only prove $(i)$ and $Z_{\alpha}(G)$, as $(i i)$ and $\chi_{\alpha}(G)$ can be proved similarly. By (4.1), we may define $f(x, y)=(x+y-2)^{\alpha}$ with $\min \{x, y\} \geq 1$ and $\max \{x, y\} \geq 2$. Suppose that $x_{1} \geq y_{1}$ and $x_{2} \geq y_{2}$. To show that $f(x, y)=(x+y-2)^{\alpha}$ is escalating, by 1.1 it suffices to show that

$$
\int_{y_{1}+x_{2}-2}^{x_{1}+x_{2}-2} \alpha t^{\alpha-1} d t \geq \int_{y_{1}+y_{2}-2}^{x_{1}+y_{2}-2} \alpha t^{\alpha-1} d t
$$

which is equivalent to

$$
\int_{y_{1}}^{x_{1}} \alpha\left(t+x_{2}-2\right)^{\alpha-1} d t \geq \int_{y_{1}}^{x_{1}} \alpha\left(t+y_{2}-2\right)^{\alpha-1} d t
$$

When $\alpha>1$ or $\alpha<0$, since $t+x_{2}-2 \geq t+y_{2}-2 \geq y_{1}+y_{2}-2 \geq 1$, we can conclude that inequality 4.2 holds. Thus, $f(x, y)$ is escalating.

When $\alpha>1$, it is easy to see that $f\left(x_{1}+1, x_{2}\right)+f\left(x_{1}+1, y_{1}-1\right)-f\left(x_{2}, y_{1}\right)-f\left(x_{1}, y_{1}\right)=\left(x_{1}+x_{2}-\right.$ $1)^{\alpha}-\left(y_{1}+x_{2}-2\right)^{\alpha} \geq 0$ holds for any $x_{1} \geq y_{1}$ and $x_{2} \geq 1\left(\right.$ since $\left.\max \left\{x_{1}, y_{1}\right\} \geq 2, \max \left\{x_{2}, y_{1}\right\} \geq 2\right)$. Furthermore, since $x+y \geq 3, g(x, y)=\frac{\partial f(x, y)}{\partial x}=\alpha(x+y-2)^{\alpha-1}>0$ and $\frac{\partial^{2} f(x, y)}{\partial x^{2}}=\alpha(\alpha-1)(x+y-$ $2)^{\alpha-2} \geq 0$. Thus, $Z_{\alpha}(G)$ is a good escalating function for $\alpha>1$.

Theorem 4.2 Let $G$ be a connected graph with at least three vertices. Then, $W_{\alpha}(G)$ is escalating for any real number $\alpha \neq 0$. Furthermore, $W_{\alpha}(G)$ is good escalating for $\alpha=1$.

Proof. Let $f(x, y)=(x y)^{\alpha}$ and $g(x, y)=\frac{\partial f(x, y)}{\partial x}$, where $\alpha \neq 0, \min \{x, y\} \geq 1$ and $\max \{x, y\} \geq 2$. For any real number $\alpha$, it is easy to see that

$$
f\left(x_{1}, x_{2}\right)+f\left(y_{1}, y_{2}\right)-f\left(x_{1}, y_{2}\right)-f\left(x_{2}, y_{1}\right)=\left(x_{1}^{\alpha}-y_{1}^{\alpha}\right)\left(x_{2}^{\alpha}-y_{2}^{\alpha}\right) \geq 0
$$

holds for any $x_{1} \geq y_{1} \geq 1$ and $x_{2} \geq y_{2} \geq 1$, and hence $W_{\alpha}(G)$ is escalating.

It is easy to check that $W_{\alpha}(G)$ is good escalating for $\alpha=1$.

Remark 4.1 Note that $W_{1}(G)=M_{2}(G)$. By Theorems 3.1 3.3 and Theorem 4.2 , we can easily deduce the main results of $9,10,19$, that is "If $\pi \triangleleft \pi^{\prime}$, then $M_{2}\left(G^{\prime}\right)>M_{2}(G)$ holds for any two extremal graphs $G \in \Gamma(\pi)$ and $G^{\prime} \in \Gamma\left(\pi^{\prime}\right)$ when $G$ is a $c$-cyclic graph for $0 \leq c \leq 2 . "$

Remark 4.2 By Theorems 2.1 and 4.2 we can conclude that an extremal graph $G$ of the Randić index or second Zagreb index among these connected graphs with fixed degree sequence is a $B F S$-tree $[9,15]$ (respectively, $U_{M}(\pi), B_{M}(\pi)$ ) when $G$ is a tree (respectively, a unicyclic graph, a bicyclic graph with $d_{n}=1[10,19)$. 
Remark 4.3 By Theorems 2.1 and 2.2, we can conclude that an extremal graph $G$ of sum-connectivity index, the third Zagreb index, reformulated Zagreb index or harmonic index among these connected graphs with fixed degree sequence is a BFS-tree (respectively, $U_{M}(\pi), B_{M}(\pi)$ ) when $G$ is a tree (respectively, a unicyclic graph, a bicyclic graph with $d_{n}=1$ ). Furthermore, Theorem 4.1 implies that Theorems 3.1 3.3 are also suitable for the third Zagreb index or reformulated Zagreb index.

Remark 4.4 Zhang et al. 22 has shown that $f(x, y)=\sqrt{\frac{x+y-2}{x y}}$ is de-escalating, and hence the results of Theorems 2.1 2.2 are also suitable for the Atom-Bond connectivity index of $G[8,17$.

Remark 4.5 By Theorem 4.1 $Z_{2}(G)$ is a good escalating function. Thus, Corollary 3.2 implies that $S_{n}^{(c)}$ is the unique extremal c-cyclic graph with maximum reformulated Zagreb indices among all c-cyclic graphs with $n \geq 4$ vertices for $0 \leq c \leq 2$. These results had been proved in 6.7 .

Acknowledgement. The authors would like to thank the referees for their valuable comments which lead to an improvement of the original manuscript.

\section{References}

[1] B. Bollobás, P. Erdös, Graphs of extremal weights, Ars Combin., 50 (1998), 225-233.

[2] E. Estrada, L. Torres, L. Rodrĺguez, I. Gutman, An atom-bond connectivity index: Modeling the enthalpy of formation of alkanes, Indian J. Chem., 37A (1998), 849-855.

[3] S. Fajtlowicz, On conjectures of Graffiti-II, Congr. Numer., 60 (1987), 187-197.

[4] I. Gutman, N. Trinajstić, Graph theory and molecular orbitals. Total $\pi$-electron energy of alternant hydrocarbons, Chem. Phys. Lett., 17 (1972), 535-538.

[5] I. Gutman, Degree-based topological indices, Croat. Chem. Acta, 86 (2013), 351-361.

[6] A. Ilić, B. Zhou, On reformulated Zagreb indices, Discrete Appl. Math., 160 (2012), 204-209.

[7] S. Ji, X. Li, B. Huo, On reformulated Zagreb indices with respect to acyclic, unicyclic and bicyclic graphs, MATCH Commun. Math. Comput. Chem., 72 (2014), 723-732.

[8] W. Lin, T. Gao, Q. Chen, X. Lin, On the minimal ABC index of connected graphs with given degree sequence, MATCH Commun. Math. Comput. Chem., 69 (2013), 571-578. 
[9] M. Liu, B. Liu, The second Zagreb indices and Wiener polarity indices of trees with given degree sequences, MATCH Commun. Math. Comput. Chem., 67 (2012), 439-450.

[10] M. Liu, B. Liu, The second Zagreb indices of unicyclic graphs with given degree sequences, Discrete Appl. Math., 167 (2014), 217-221.

[11] A.W. Marshall, I. Olkin, Inequalities: Theory of Majorization and its Applications, Academic Press, New York, 1979.

[12] A. Miličević, S. Nikolić, N. Trinajstić, On reformulated Zagreb indices, Mol. Diversity, 8 (2004), 393-399.

[13] M. Randić, On characterization of molecular branching, J. Amer. Chem. Soc., 97 (1975), 66096615.

[14] G.H. Shirdel, H. Rezapour, A.M. Sayadi, The hyper-Zagreb index of graph operations, Iran. J. Math. Chem., 4 (2013), 213-220.

[15] H. Wang, Extremal trees with given degree sequence for the Randić index, Discrete Math., 308 (2008), 3407-3411.

[16] H. Wang, Functions on adjacent vertex degrees of trees with given degree sequence, Cent. Eur. J. Math., 12 (2014), 1656-1663.

[17] R. Xing, B. Zhou, Extremal trees with fixed degree sequence for atom-bond connectivity index, Filomat, 26 (2012), 683-688.

[18] K. Xu, K.C. Das, S. Balachandran, Maximizing the Zagreb indices of $(n, m)$-graphs, MATCH Commun. Math. Comput. Chem., 72 (2014), 641-654.

[19] W.-G. Yuan, X.-D. Zhang, The second Zagreb indices of graphs with given degree sequences, Discrete Appl. Math., 185 (2015), 230-238.

[20] G. Zhang, Y. Chen, Functions on adjacent vertex degrees of graphs with prescribed degree sequence, MATCH Commun. Math. Comput. Chem., 80 (2018), 129-139. 
[21] X.-D. Zhang, The Laplacian spectral radii of trees with degree sequences, Discrete Math., 308 (2008), 3143-3150.

[22] X.-M. Zhang, X.-D. Zhang, R. Bass, H. Wang, Extremal trees with respect to functions on adjacent vertex degrees, MATCH Commun. Math. Comput. Chem., 78 (2017), 307-322.

[23] B. Zhou, N. Trinajstić, On a novel connectivity index, J. Math. Chem., 46 (2009), 1252-1270.

[24] B. Zhou, N. Trinajstić, On general sum-connectivity index, J. Math. Chem., 47 (2010), 210-218. 